\section{H. Venner}

\section{W. E. ten Napel}

\section{R. Bosma}

The University of Twente, Enschede, The Netherlands

\section{Advanced Multilevel Solution of the EHL Line Contact Problem}

The application of multilevel multi-integration to the calculation of the elastic deformation integrals and the use of an alternative relaxation process in the multilevel solution of the governing equations have resulted in an algorithm solving the EHL line contact problem in $\mathrm{O}(\mathrm{n} \ln \mathrm{n})$ operations, also for highly loaded situations. The reduction in computing time thus obtained was used to solve the problem using large numbers of nodal points and to study the pressure spike. The presented algorithm will enable fast and accurate solution of surface roughness and transient problems.

\section{Introduction}

Over the years, following among others Petrusevich [1] and Dowson and Higginson [2], Elasto Hydrodynamic Lubrication (EHL) has received much attention in tribological literature. This extensive interest in both EHL line- and point contact problems has resulted in several numerical algorithms of varying efficiency for solving these problems. Most of the papers deal with the line contact problem and the most widely used numerical solution method is a system Newton Raphson approach [3-7]. One of the disadvantages of this method with respect to the solution of the EHL problem is that the complexity is $O\left(n^{3}\right)$; if $n$ is the number of calculational points. Because of the elastic deformations, the Jacobian matrix is a full $n \times n$ matrix, and inverting it requires $O\left(n^{3}\right)$ operations. For large $n$, i.e. for point contact problems, this will lead to rather excessive computing times. This problem was partly overcome by using large mainframe and super computers and local grid refinement techniques. Another disadvantage of the method is that, with increasing load, the Jacobian matrix becomes almost singular, making the solution of highly loaded situations rather cumbersome. Finally, a straightforward extension of a Newton Raphson algorithm to point contact problems is not possible because of the cavitation condition, see [4]. Improvements regarding both complexity and stability have been reported. For instance, Houpert and Hamrock [6] managed to obtain solutions for high loads, and recently Chang et al. [8] reduced the complexity to $O\left(n^{2}\right)$ by truncating the Jacobian matrix to a tridiagonal matrix, discarding the terms that reflect the global relation between filmthickness and pressure caused by the elastic deformation. However, since these terms become increasingly important for higher loads, the truncation can not alleviate the difficulties of solving the highly loaded situations.

An alternative method of solution was introduced by Lubrecht et al. [9-11]. They employed a Gauss Seidel relaxation process to solve the equations together with multilevel techniques to accelerate convergence. The complexity of their multilevel algorithm was $O\left(n^{2}\right)$ mainly caused by the calculation of the elastic deformation integrals (see also section 4). The reduction in computing time, enabled them to solve both

Contributed by the Tribology Division for publication in the Journal of Tribology. Manuscript received by the 'Tribology Division February 24,' 1989. line- and point contact problems with a rather large number of nodes on a relatively small computer (VAX 11/750). Solutions up to a maximum Hertzian pressure of $\pm 2.0 \mathrm{GPa}$ have been presented. However, with increasing load, underrelaxation and local relaxation with local film thickness updates were needed to obtain a stable relaxation process.

This paper describes the development of a new relaxation process for solving the EHL line contact equations. No local updates or recalculations of film thicknesses are needed and also large underrelaxation factors can be avoided. Furthermore, the complexity of the calculation of the elastic deformation integrals was reduced from $O\left(n^{2}\right)$ to $O(n \ln n)$ by using a newly developed multilevel technique "multilevel multiintegration." The result is an algorithm solving the EHL line contact problem up to maximum Hertzian pressures well over $4.0 \mathrm{GPa}$ in $O(n \ln n)$ operations. With the presented algorithm the EHL line contact problem can be solved using over 30.000 calculational points in a reasonable computing time on a mini computer. This allows a detailed study of characteristic features such as the pressure spike in many situations. Furthermore, it will enable the fast solution of transient and surface roughness problems.

\section{Equations}

All equations are made dimensionless using the Hertzian dry contact parameters. (see nomenclature) The Reynolds equation then reads:

$$
\frac{d}{d X}\left(\epsilon \frac{d P}{d X}\right)-\frac{d(\bar{\rho} H)}{d X}=0
$$

with the cavitation condition: $P \geq 0$.

$\epsilon$ is given by:

$$
\epsilon=\frac{\bar{\rho} H^{3}}{\bar{\eta} \lambda}
$$

where

$$
\lambda=\frac{6 \eta_{o} u_{s} R^{2}}{b^{3} p_{h}}
$$


The dimensionless lubricant density $\bar{\rho}$ is assumed to depend on the pressure according to the Dowson and Higginson relation [2]. Moreover, the Roelands viscosity pressure relation is used. It reads:

$$
\bar{\eta}=\frac{\eta}{\eta_{o}}=\exp \left[\frac{\alpha p_{o}}{z}\left[\left(1+\frac{P}{P_{o}}\right)^{z}-1\right]\right]
$$

where $P_{o}=p_{o} / p_{h}$ and $p_{o}=1.9810^{8}\left[\mathrm{Nm}^{-2}\right]$

Using second order central discretization for the first term of (1) and first order upstream discretization of the wedge term, the discretized Reynolds equation in node $i$ on a grid with mesh size $\Delta$ reads:

$$
\begin{aligned}
& \Delta^{-2}\left(\epsilon_{i-1 / 2} P_{i-1}-\right.\left.\left(\epsilon_{i-1 / 2}+\epsilon_{i+1 / 2}\right) P_{i}+\epsilon_{i+1 / 2} P_{i+1}\right) \\
&-\Delta^{-1}\left(\bar{\rho}_{i} H_{i}-\bar{\rho}_{i-1} H_{i-1}\right)=0
\end{aligned}
$$

with the cavitation condition $P_{i} \geq 0$.

Similarly the dimensionless film thickness equation reads:

$$
H(X)=H_{o}+\frac{X^{2}}{2}-\frac{1}{\pi} \int_{X_{a}}^{X_{b}} P(Y) \ln |X-Y| d Y
$$

Discretization results in:

$$
H_{i}=H_{o}+\frac{X_{i}^{2}}{2}-\frac{1}{\pi} \sum_{j=1}^{n} K_{i j}^{\Delta \Delta} P_{j}
$$

where

$$
\begin{aligned}
K_{i j}^{\Delta \Delta} & =\left(i-j+\frac{1}{2}\right) \Delta\left(\ln \left(\left|i-j+\frac{1}{2}\right| \Delta\right)-1\right) \\
& -\left(i-j-\frac{1}{2}\right) \Delta\left(\ln \left(\left|i-j-\frac{1}{2}\right| \Delta\right)-1\right)
\end{aligned}
$$

The dimensionless force balance equation, stating that the integral over the pressure should equal the externally applied load, reads after discretization:

$$
\Delta \sum_{j=1}^{n-1} \frac{\left(P_{j}+P_{j+1}\right)}{2}-\frac{\pi}{2}=0
$$

\section{Multigrid Method}

The three discretized equations (3), (5), and (6) are simultaneously solved using multilevel, i.e. multigrid, techniques. For a description of the multigrid method applied to
EHL problems the reader is referred to Lubrecht et al. [9-11]. For more information on multigrid techniques in general the reader is referred to Brandt [12]. An introduction to multigrid techniques is given in [13]. In this paper only the relaxation process, which is in fact the basis of the algorithm, is discussed in detail in section 5 .

\section{Multilevel Multi-Integration}

Recently Brandt and Lubrecht [14], [15] developed a multilevel algorithm for the fast calculation of integrals of the type of the elastic deformation integrals in equation (4). As can be seen from equation (5) the calculation of the elastic deformation in one point requires $O(n)$ operations and thus the calculation of all $H_{i}$ costs $O\left(n^{2}\right)$ operations. Using multilevel multi-integration computing time can, without loss of accuracy, be reduced from $O\left(n^{2}\right)$ to $O(n \ln n)$, see [14]. For large $n$ this is approximately $O(n)$ since $\ln (n)$ increases fairly slow with $n$. This technique was implemented in the multilevel solver of equations (3), (5), and (6). It is extensively described in [14] and [15] and will not be discussed here.

\section{Relaxation Process}

One of the problems encountered in the numerical solution of the EHL line contact problem is that the coefficient $\epsilon$ in (1) varies several orders of magnitude over the calculational domain. In both the inlet and the outlet region $\epsilon<<1$ since in those regions $\bar{\eta}$ is small and $H^{3}$ large. On the contrary, in the contact region $\bar{\eta}$ is very large and $H^{3}$ is relatively small and thus $\epsilon<<1$. The relaxation process employed in a multilevel solver of the equations (3), (5), and (6) should be a good error smoother for both large and small values of $\epsilon$. The performance of the multilevel algorithm (incorporating this relaxation process) is determined by the efficiency of this relaxation to reduce the amplitude of error components with wavelengths of the order of the mesh size. Regarding the low frequency components of the error, the only demand the relaxation process should meet is that they are not amplified, otherwise the process will be unstable. The amplitude reduction factor of those components is in fact not very important since they are solved on the coarser grids.

A new relaxation process for the EHL line contact problem was developed based on the results obtained from the analysis of some model problems resembling the full problem. The first problem studied was the following:

Nomenclature

$b=$ half-width Hertzian contact region, $b=\sqrt{\frac{8 w R}{\pi \mathrm{E}^{\prime}}}$

$E^{\prime}=$ reduced modulus of elasticity,

$$
\frac{2}{\mathrm{E}^{\prime}}=\frac{1-\nu_{1}^{2}}{\mathrm{E}_{1}}+\frac{1-\nu_{2}^{2}}{\mathrm{E}_{2}}
$$

$\mathrm{E}=$ elasticity modulus (Young's modulus)

$G=$ material parameter, $G=\alpha \mathrm{E}^{\prime}$

$H=$ dimensionless film thickness,

$H=\frac{h R}{b^{2}}$

$$
\begin{aligned}
& H_{o}= \begin{array}{l}
\text { integration constant in } \\
\text { dimensionless film } \\
\text { thickness equation }
\end{array} \\
& H_{\min }=\begin{array}{l}
\text { dimensionless minimum } \\
\text { film thickness (Moes) }
\end{array} \\
& H_{\min }=\frac{h_{\min }}{R}(2 U)^{-1 / 2} \\
& H_{m}= \text { dimensionless minimum } \\
& \text { film thickness } \\
& H_{m}=\frac{h_{\min } R}{b^{2}} \\
& h_{\min }= \text { minimum film thickness } \\
& h= \text { film thickness } \\
& K_{|i-j|}= \text { discretized kernel in film } \\
& \text { thickness equation }
\end{aligned}
$$

$$
\begin{aligned}
L= & \text { dimensionless material } \\
& \text { parameter (Moes) } \\
& L=G(2 U)^{1 / 4} \\
M= & \text { dimensionless load } \\
& \text { parameter (Moes) } \\
& M=W(2 U)^{-1 / 2} \\
n= & \text { number of calculational } \\
\text { points } & \text { proportional to } n \\
O(n)= & \text { dimensionless pressure, } \\
P= & \begin{array}{l}
p \\
p h
\end{array} \\
P_{s}= & \text { dimensionless spike height } \\
p= & \text { pressure } \\
p_{h}= & \text { maximum Hertzian } \\
& \text { pressure } \\
& p_{h}=\frac{2 w}{\pi b}
\end{aligned}
$$




$$
\frac{d}{d X}\left(\epsilon \frac{d P}{d X}\right)-\frac{d H}{d X}=0
$$

where $H$ is given by equation (4) and $\epsilon$ was initially assumed to be a constant. This equation is in fact a linearized version of the Reynolds equation. Discretization of the equation results in:

$$
\begin{gathered}
\Delta^{-1}\left(\epsilon_{i-1 / 2} P_{i-1}-\left(\epsilon_{i-1 / 2}+\epsilon_{i+1 / 2}\right) P_{i}+\epsilon_{i+1 / 2} P_{i+1}\right) \\
-\left(H_{i}-H_{i-1}\right)=0
\end{gathered}
$$

In case of constant $\epsilon$ this equation reduces to:

$$
\frac{\epsilon}{\Delta}\left(P_{i-1}-2 P_{i}+P_{i+1}\right)-\left(H_{i}-H_{i-1}\right)=0
$$

Since this equation is linear, a smoothing rate analysis can be carried out for different types of relaxation. For detailed information on smoothing rate analysis the reader is referred to [12]. In case of this model problem the smoothing rate, i.e., the amplitude reduction factor for an error component with a certain wavelength, depends on $\epsilon / \Delta$, and is thus grid dependent. To measure the performance of the relaxation in a multilevel algorithm the asymptotic smoothing rate is used. This is the maximum of all smoothing rates for the high frequency components. This asymptotic smoothing rate determines the error reduction that can be obtained in one coarse grid correction cycle ( $V$ or $W$ cycle, see [12]). Two relaxation types were considered in particular. The first is simple Gauss Seidel relaxation on $P$ without local updates or recalculations of film thicknesses. This relaxation is very well suited to solve the problem for large values of $\epsilon / \Delta$, resulting in an asymptotic smoothing rate of 0.5 . This is no surprise since for large values of $\epsilon / \Delta$ the equation resembles the one dimensional Poisson equation, because the second term in equation (9) becomes negligible compared with the first term. For small values of $\epsilon / \Delta$ however, low frequency components of the error are no longer reduced and the Gauss Seidel relaxation diverges. Local recalculation of some filmthicknesses in the vicinity of the point where the pressure is changed does not alter this behavior. The lower limit of the stability range is approximately $\epsilon / \Delta=0.01$. However, on very coarse grids the relaxation may even be unstable for larger values of $\epsilon / \Delta$.

A Jacobi dipole relaxation on the contrary, is very well suited to solve the problem for small values of $\epsilon / \Delta$ and even for $\epsilon=0$ (asymptotic smoothing rate 0.4 ). Instead of changing only $P_{i}$ to satisfy the discrete equation in gridpoint $i$ as in the simple Gauss Seidel relaxation, changes $\delta_{i}$ and $-\delta_{i}$ are calculated for respectively $P_{i}$ and $P_{i-1}$. All $P_{i}$ 's are simultaneously adjusted at the end of the relaxation sweep. This relaxation process becomes unstable for larger values of $\epsilon / \Delta$. If an underrelaxation factor of 0.6 is used the relaxation is stable even for large values of $\epsilon / \Delta$. For values of $\epsilon / \Delta$ where both relaxation types are stable, the Gauss-Seidel relaxation generally outranks the Jacobi dipole relaxation.

Using the overlap in stability regions of both relaxation processes, provided an underrelaxation factor 0.6 is employed in the Jacobi dipole relaxation, an efficient multilevel algorithm solving equation (9) for all values of $\epsilon$ was developed. On grids where $\epsilon / \Delta<0.01$, generally the coarsest grids, the Jacobi dipole relaxation was employed, whereas on grids where $\epsilon / \Delta \geq 0.01$ the simple Gauss Seidel relaxation was used.

The next step was allowing $\epsilon$ to vary over the domain and thus to develop an algorithm solving equation (8) for different functions $\epsilon(X)$. For instance $\epsilon(X)$ was chosen:

$$
\epsilon(X)=\left\{\begin{array}{l}
X^{6} \text { if }|X|>1 \\
0 \text { otherwise }
\end{array}\right.
$$

A function that resembles the $\epsilon$ occurring in equation (1). In the multilevel algorithm described above, the relaxation type used on a specific grid depended on the value of $\epsilon / \Delta$ on that grid. Since relaxation is, by its nature, a local process, both relaxation types can also be combined on one grid. Thus, in regions of the domain where $\epsilon / \Delta \geq 0.01$ simple Gauss Seidel changes are applied, whereas in regions where $\epsilon / \Delta<0.01$ Jacobi dipole changes are calculated and applied when the sweep is completed. Using this hybrid relaxation type in a multilevel solver for equation (9) with $\epsilon(X)$ given by (10) a reduction of the residuals of one order of magnitude per coarse grid correction cycle ( $\mathrm{V}(2,2)$ or $\mathrm{W}(2,2)$ cycle) was easily obtained.

Finally this hybrid relaxation process was successfully implemented in a multilevel solver of the full nonlinear EHL line contact problem. Because of the nonlinearity, an underrelaxation factor varying from 0.5 to 1.0 was used for the Gauss Seidel changes and similarly an underrelaxation factor varying from 0.3 to 0.6 (the aforementioned upper limit) for the Jacobi dipole changes. The relaxation process was found to be stable up to maximum Hertzian pressures well over 4.0 GPa.

\section{Calculational Details}

Most solutions presented in this paper have been calculated Nomenclature (cont.)

$$
\begin{aligned}
& p_{o}=\text { constant in Roelands' } \\
& \text { relation } \\
& P_{o}=\frac{p_{o}}{p_{h}} \\
& R=\text { reduced radius of } \\
& \text { curvature } \\
& U=\text { dimensionless speed } \\
& \text { parameter, } \\
& 2 U=\frac{\eta_{o} u_{s}}{E^{\prime} R} \\
& u_{s}=\text { sum velocity } \\
& W=\text { dimensionless load, } \\
& W=\frac{w}{E^{\prime} R} \\
& w=\text { load per unit width } \\
& X=\text { dimensionless coordinate, } \\
& X=\frac{x}{b} \\
& x=\text { coordinate } \\
& z=\text { Roelands' pressure } \\
& \text { viscosity parameter } \\
& \bar{\alpha}=\text { dimensionless pressure } \\
& \begin{array}{l}
\alpha=\text { pressure viscosity index } \\
\Delta=\text { distance between two }
\end{array} \\
& \text { neighboring gridpoints } \\
& \epsilon=\text { coefficient in equation } \\
& \lambda=\text { dimensionless velocity } \\
& \text { parameter, } \\
& \lambda=6 \frac{\eta_{o} u_{s} R^{2}}{b^{3} p_{h}}
\end{aligned}
$$


Table 1 Computing time in hours:minutes:seconds as a function of the number of nodes. (HP 9000/800 computer)

\begin{tabular}{rrrr}
\hline level & $\mathrm{n}$ & 2V-cycles & 2W-cycles \\
\hline 5 & 225 & 12 & 25 \\
\hline 6 & 449 & 24 & 58 \\
\hline 7 & 897 & 48 & $2: 18$ \\
\hline 8 & 1793 & $1: 38$ & $5: 18$ \\
\hline 9 & 3585 & $3: 17$ & $11: 44$ \\
\hline 10 & 7169 & $6: 38$ & $27: 45$ \\
\hline 11 & 14337 & $13: 20$ & $58: 30$ \\
\hline 12 & 28673 & $26: 50$ & $2: 04: 00$ \\
\hline 13 & 57345 & $1: 03: 00$ & $5: 04: 00$ \\
\hline 14 & 114689 & $2: 08: 00$ & $10: 45: 00$ \\
\hline
\end{tabular}

EHL Line Contact.

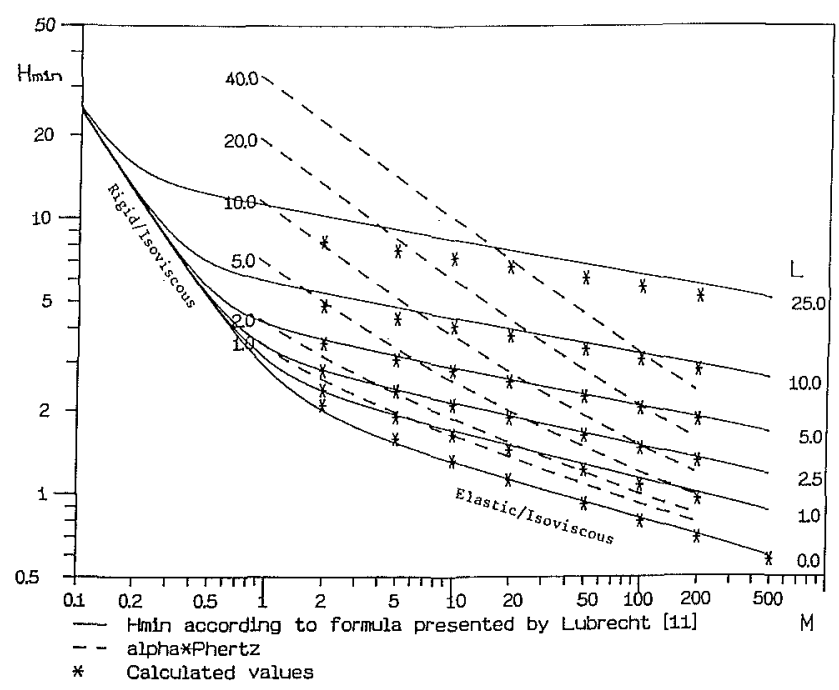

Fig. 1 The calculated value of the Moes dimensionless minimum film thickness number (") as a function of $M$ and $L$. The solid lines give the predicted values according to the formula presented by Lubrecht [11]. The dashed lines are lines of constant $\bar{\alpha}$.

on a domain extending from $X_{a}=-4$ to $X_{b}=1.5$. In some low load situations however, a larger inlet area was used to avoid numerically starved lubrication whereas in highly loaded situations a smaller inlet area was sufficient. A Full Multi Grid algorithm [12] with $2 \mathrm{~V}$ cycles per refinement gave converged solutions up to the level of the truncation error for low load situations. With increasing load the solution of the force equilibrium equation becomes more difficult. This equation is in fact a global boundary condition and is only relaxed on the coarsest grid (by adjusting $H_{o}$ ) [9], [12]. Thus for higher loads the coarsest grid should be visited more often. In those situations $2 \mathrm{~W}$ cycles were used. In all cases the coarsest grid employed in the $\mathrm{V}$ or $\mathrm{W}$ cycle consisted of 15 nodes. Convergence to the level of the truncation error was checked as described in [9] and [10]. Calculation times as a function of the number of nodes for Full Multi Grid with either $2 \mathrm{~V}$ or $2 \mathrm{~W}$ cycles per refinement [12], are given in Table 1 . The results presented in this table clearly demonstrate the $O(n \ln n)$ complexity of the algorithm. The larger increase going from level 12 to 13 is caused by the change from a 6 th order to a 10th order transfer operator in the multilevel calculation of the elastic deformation integrals, in order to keep the additional error introduced by the multilevel calculation small compared to the discretization error. All calculations have been carried out on a HP $9000 / 800$ computer.

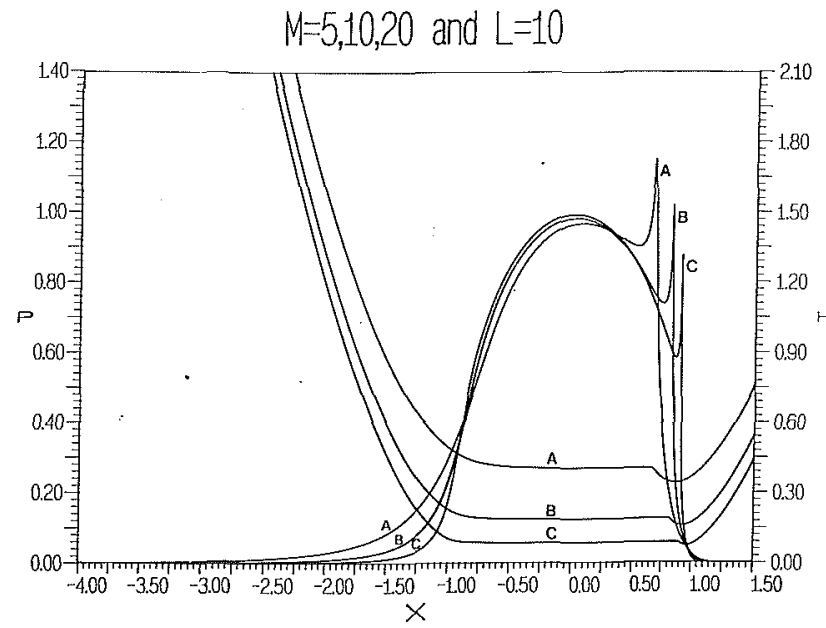

Fig. 2 Pressure distribution and film thickness for $M=5, L=10$ (A), $M=10, L=10(B)$, and $M=20, L=10(C)$

\section{Results}

All results are presented in terms of the Moes dimensionless parameters. The calculated dimensionless minimum filmthickness number $H_{\min }$ as a function of the load parameter $M$ and material parameter $L$ is presented in Fig. 1. Several asymptotic solutions, known from literature, can be drawn in this plot. These asymptotic solutions are: the rigid-isoviscous asymptote, describing the solid as nondeformable and the fluid as being of constant viscosity, calculated by Martin [16] and Gümbel [17]. Secondly the elastic-isoviscous asymptote, taking the elastic deformation of the solids into account, but the fluid remains of constant viscosity [18]. Lubrecht [11] presented a formula for $H_{\min }$ as a function of $M$ and $L$. The formula incorporates the aforementioned asymptotic solutions. The solid lines in Fig. 1, give the predictions based on this formula. For reasons of comparison, the dashed lines are lines of constant $\bar{\alpha}$. Assuming $\alpha=1.7 \times 10^{-8}\left[\mathrm{~Pa}^{-1}\right]$, solutions have been calculated up to a maximum Hertzian pressure of $\pm 8.0 \mathrm{GPa}$. It should be noted that these extreme situations are of little practical importance and are only presented to demonstrate the stability of the algorithm.

Pressure distribution and film thickness for $L=10$ and three different values of $M$ are shown in Fig. 2. Blowups of the pressure distribution in the spike region for these situations are presented in Fig. 3. The number of calculation points in the parts of the domain presented are given below the figures. Figure 4 shows pressure distribution and filmthickness, together with a blow up of the pressure distribution in the spike region, for a much higher load, i.e., $M=200$ and $L=$ 8 . In all four situations presented the number of nodes used is large enough to describe the pressure distribution accurately, even in the spike region. The results clearly show that in these situations the spike is smooth, provided it is studied at a sufficiently small length scale, see also section 8 .

Since a first order upstream discretization was used for the wedge term in equation (1), all characteristic results such as the minimum film thickness $H_{m}$, and the pressure spike height $P_{s}$, converged in most situations first order to a limiting value with successively halving the mesh width. For $M=20$ and $L$ $=10$ this convergence is illustrated in table 2 .

\section{The Pressure Spike}

One of the most interesting phenomena in EHL is the occurrence of a second local maximum in the pressure distribution located near the outlet. It is generally referred to as the "pressure spike" and was firstly reported by Petrusevich [1]. Although it is generally accepted that this spike is caused by 
Detail spike (A)

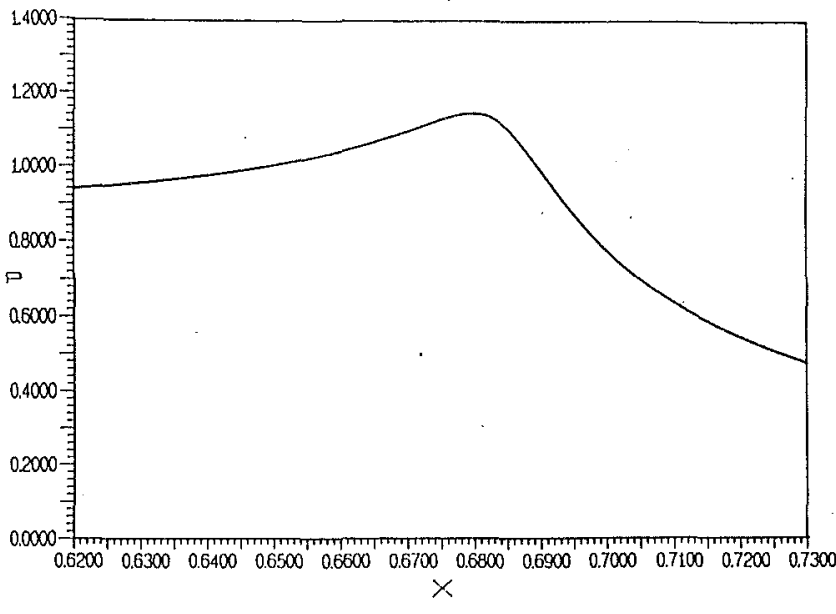

Fig. 3A Blowup of the pressure distribution in the spike region for $M=5, L=10.573$ nodes in presented region

Detail spike (B)

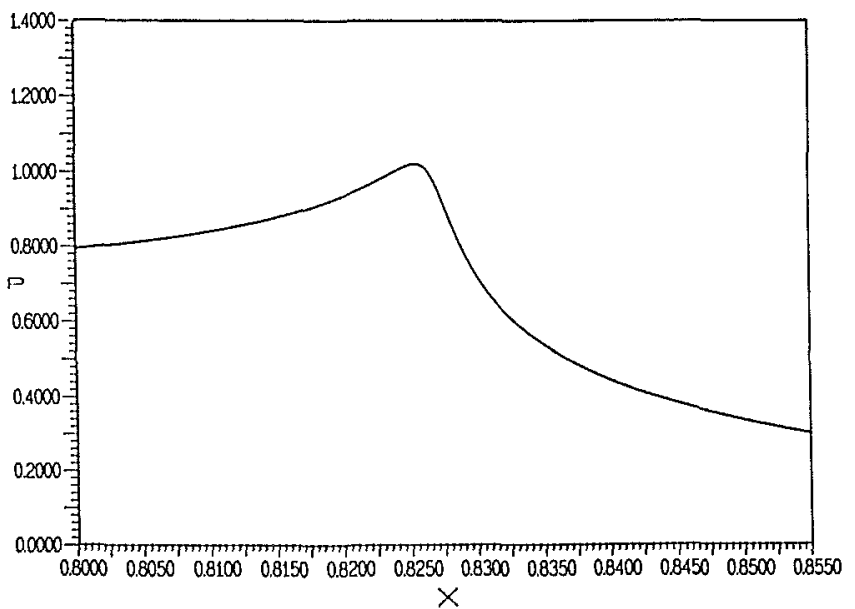

Fig. 3B Blowup of the pressure distribution in the spike region for $M=$ $10, L=10.286$ nodes in presented region

\section{Detail spike (C)}

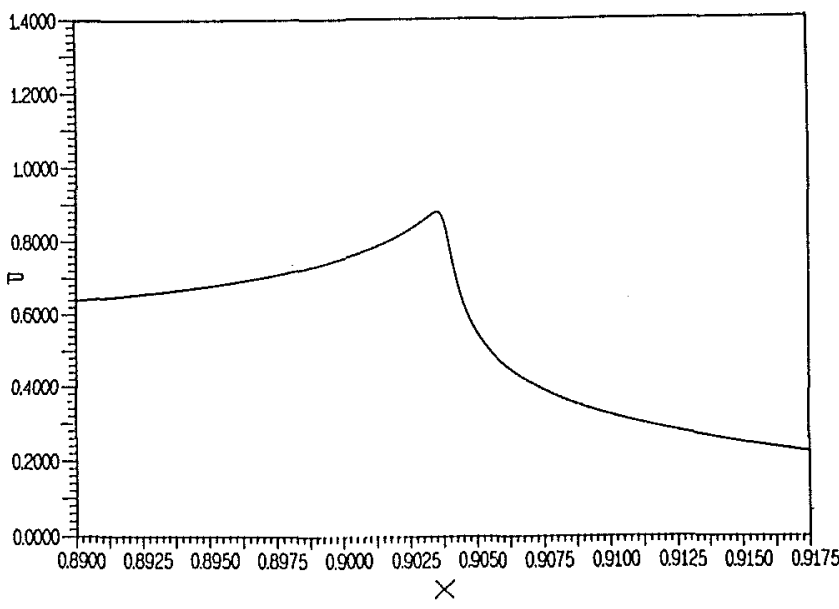

Fig. 3C Blowup of the pressure distribution in the spike region for $M=20, L=10.573$ nodes in presented region

the exponential relation between the viscosity and the pressure [9], [19] (no spike is found in case of an isoviscous lubricant), its height has been the subject of many discussions. See for example [7], [9], [21], and references therein.

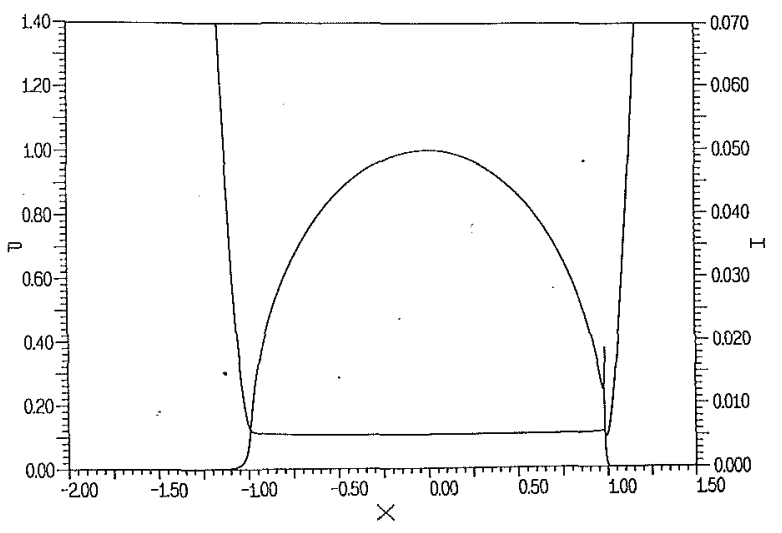

Fig. 4(a) Pressure distribution and film thickness for $M=200, L=8$

Detail spike

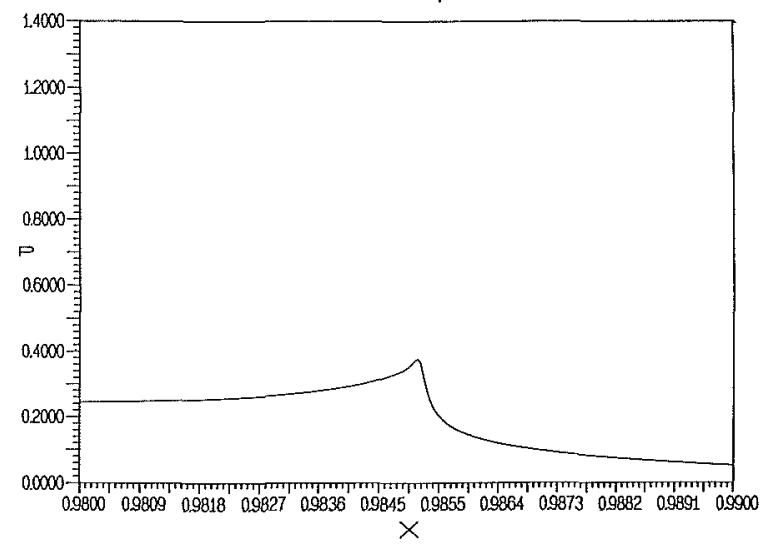

Fig. $4(b)$ Blowup of the pressure distribution in the spike region for $M=200$ and $L=8.208$ nodes in presented region

Table 2 Convergence of spike height and minimum filmthickness with increasing number of nodes for $M=20$ and $L=10$

\begin{tabular}{rrrr}
\hline level & $\mathrm{n}$ & $H_{m}$ & $P_{s}$ \\
\hline 6 & 449 & 0.0750 & 0.677 \\
\hline 7 & 897 & 0.0744 & 0.710 \\
\hline 8 & 1793 & 0.0741 & 0.758 \\
\hline 9 & 3585 & 0.0739 & 0.787 \\
\hline 10 & 7169 & 0.0738 & 0.825 \\
\hline 11 & 14337 & 0.0737 & 0.850 \\
\hline 12 & 28673 & 0.0737 & 0.867 \\
\hline 13 & 57345 & 0.0737 & 0.875 \\
\hline 14 & 114689 & 0.0737 & 0.879 \\
\hline
\end{tabular}

Since the newly developed algorithm enables the use of large nodal densities, the spike can be captured in many situations. Both occurrence and height of the spike as a function of the governing parameters were investigated. The results of this study are reported in [20]. The parameter range where a spike can be expected and the effect of the compressibility and of the viscosity pressure relation applied, on this parameter range, as well as results of spike height calculations for both an incompressible and compressible lubricant, are presented.

The variation of the spike height with the governing parameters observed for the situation considered in this paper, i.e., using the Roelands equation and assuming a compressible 
Spike Height

(Roelands/compressible)

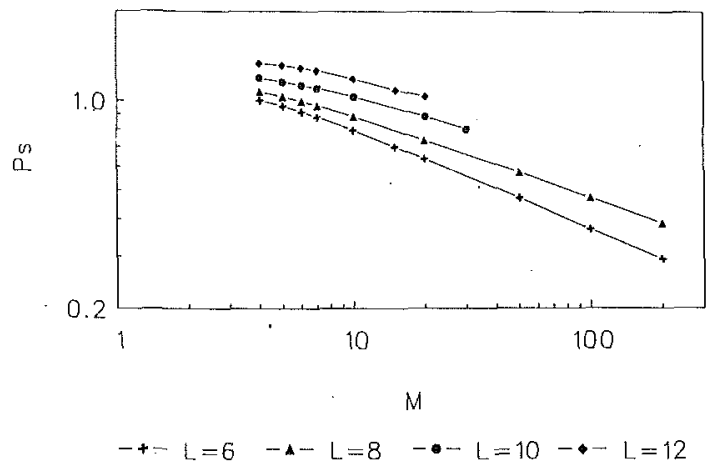

Fig. 5 The calculated dimensionless splke height $P_{s}$ as a function of $M$ for some values of $L$

$$
\begin{aligned}
& \text { Spike Height } \\
& \text { (roelonds/compressible) }
\end{aligned}
$$

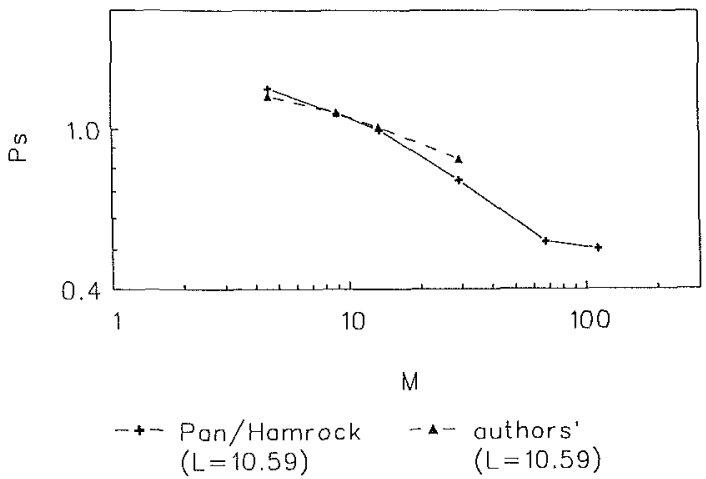

Fig. 6 The calculated dimensionless splke height for $L=10.59$ as a function of $M$ compared with results presented by Pan and Hamrock [21]

lubricant, is shown in Fig. 5. Only situations where, within the current limitations of both computing time and computer storage, convergence of the spike height with increasing number of nodes could be shown, are displayed. To obtain results beyond this parameter range, local grid refinement techniques are recommended. Figure 5 shows that, within the parameter range presented, the spike height decreases with increasing load. A behavior that can also be observed from Fig. 3. Since convergence of the spike height with increasing number of nodes was checked this decrease is not a numerical effect.

Finally, Fig. 6 compares the spike heights calculated in a number of situations with results presented by Pan and Hamrock [21] for the same conditions. Although their data show a somewhat steeper decrease of the spike height with increasing load, the two sets of results agree reasonably well. Part of the differences observed might be caused by the use of different values of the parameters $\alpha$ and $z$ in the Roelands equation.

\section{Conclusions}

An alternative relaxation process for the EHL line contact was developed. The use of the recently developed multilevel technique "multilevel multi-integration" in the calculation of the elastic deformation integrals and common multigrid techniques to accelerate convergence of the relaxation process, have resulted in an algorithm solving the EHL line contact problem in $O(n \ln n)$ operations. Solutions up to maximum
Hertzian pressure well over $4.0 \mathrm{GPa}$ have been obtained. The complexity $O(n \ln n)$ makes it possible to solve the problem using large numbers of calculational points in a reasonable computing time on a mini computer, as well as to study the pressure spike. The algorithm presented can be extended to point contact situations and will enable fast and accurate solution of transient and surface roughness problems.

\section{Acknowledgment}

The authors would like to thank Prof. A. Brandt and Dr. A. A. Lubrecht for the many helpful discussions. Part of the work was done during a stay at the Weizmann Institute of Science, Rehovot, Israel, sponsored by the faculty of Mechanical Engineering of the University of Twente. The research was also sponsored by SKF Engineering and Research Center B.V., Nieuwegein, The Netherlands.

\section{References}

1 Petrusevich, A. I., "Fundamental Conclusions from the Contact Hydrodynamic Theory of Lubricaton." Izv. Akad. Nauk SSR (OTN), Vol. 2, 1951, pp. 209-223.

2 Dowson, D. J., and Higginson, G. R., Elasto-Hydrodynamic Lubrication, Oxford, Pergamon, Ist edition, 1966.

3 Okamura, H., "A Contribution to the Numerical Analysis of Isothermal Elasto-Hydrodynamic Lubrication," Proceedings 1982 Leeds-Lyon Symposium on Tribology, 1982, pp. 313-320.

$4 \mathrm{Oh}$, K. P., "The Numerical Solution of Dynamically Loaded Elastohydrodynamic Contact as a Non-Linear Complementarity Problem," ASME Journal of Tribology, Vol. 106, 1984, pp. 88-95.

5 Kostreva, M. M., "Elasto Hydrodynamic Lubrication: A Non-Linear Complementarity Problem," Int. Journal for Numerical Methods in Fluids, Vol. 4, 1984, pp. 377-397.

6 Houpert, L. G., and Hamrock, B. J., "Fast Approach for Calculating Film Thicknesses and Pressures in Elastohydrodynamically Lubricated Contacts at High Loads," ASME Journal of Tribology, Vol. 108, 1986, pp. 411-420.

7 Bisset, E. J., and Glander, D. W., "A Highly Accurate Approach That Resolves the Pressure Spike of Elastohydrodynamic Lubrication," ASME Journal of Tribology, Vol. 110, 1988, pp. 241-246.

8 Conry, T. F., Chang, L., and Cusano, C., "An Efficient, Robust, MultiLevel Computational Algorithm for Elastodynamic Lubrication," ASME JouRNAl of Trubology, Vol. 111, 1988, pp. 193-199.

9 Lubrecht, A. A., "The Numerical Solution of the Elastohydrodynamically Lubricated Line- and Point Contact Problem Using Multigrid Techniques," PhD. thesis, University of Twente, Enschede, The Netherlands, 1987.

10 Lubrecht, A. A., Ten Napel, W. E., and Bosma, R., "Multigrid, an Alternative Method for Calculating Film Thickness and Pressure Profiles in Elastohydrodynamically Lubricated Line Contacts," ASME Journal, of Tribology, Vol. 108, 1986, pp. 551-556.

11 Lubrecht, A. A., Breukink, G. A. C., Moes, H., Ten Napel, W. E., and Bosma, R. "Solving Reynolds Equation for E.H.L. Line Contacts by Application of a Multigrid Method," Proceedings of the 13th Leeds-Lyon Symposium on Tribology, 1986, pp. 175-182.

12 Brandt, A., "Multigrid Techniques: 1984 Guide, With Applications to Fluid Dynamics," Gesellschaft für Mathematik und Datenverarbeitung MBH Bonn, 1984

13 Briggs, W. L., “A Multigrid Tutorial,” $S I A M, 1988$.

14 Brandt, A., and Lubrecht, A. A., "Multi-Level Multi Integration and Fast Solution of Integral Equations," to be published in Journal of Computational Physics, 1988.

15 Lubrecht, A. A., and Ioannides, E., "A Fast Solution of the Dry Contact Problem and the Associated Sub-Surface Stress Field, Using Multilevel Techniques," to be presented at the 1989 ASME/STLE Tribology conference, Oct. 16-19, 1989, Fort Lauderdale, Fla.

16 Martin, H. M., "Lubrication of Gear Teeth," Engineering, Vol. 102, 1916, pp. 119-121.

17 Gümbel, L., “Über geschmierte Arbeitsräder," Z. ges. Turbinewesen, Vol. 13,1916, p. 357.

18 Moes, H., "On Survey Diagrams," Internal report, Laboratory of Tribology, University of Twente, The Netherlands, 1985.

19 Hamrock, B. J., Ping Pan, and Rong-Tsong Lee, "Pressure Spikes in Elastohydrodynamically Lubricated Conjunctions," ASME JouRNAL OF Tribology, Vol. 110, 1988, pp. 279-284.

20 Venner, C. H., and Ten Napel, W. E., "Numerical Calculations of the Pressure Spike in EHL," Proceedings EUROTRIB, Vol. 2, 1989, pp. 196-201.

21 Ping Pan, and Hamrock, B. J., "Simple Formulas for Performance Parameters in Elastohydrodynamically Lubricated Line Contacts," ASME Journal of Tribology, Vol. 111, 1988, pp. 246-251. 


\section{DIS CUSSIO N}

\section{R. Verstappen ${ }^{1}$}

The authors deal with the problem of computing the deflection of a surface caused by a pressure loading. In addition, an alternative relaxation procedure is introduced in the multilevel algorithm for solving Reynolds' equation. Both the new integration and relaxation techniques are of a high standard. Yet, it seems that these advanced techniques are applied in solving physical unrealistic problems, using needless dense and uniform grids.

With respect to the grid it may be observed that taking $10^{+5}$ or more gridpoints for a contact of 0.01 to $0.1 \mathrm{~mm}$. gives a stepsize of a few Angström. Phenomenon on such scales is certainly not described by Reynolds' equation. So, why is the grid that dense?

Taking the parameter setting as in the paper, i.e., alfa $=1.7$ $10^{-8} \mathrm{~Pa}^{-1}, p_{0}=1.9810^{+8} \mathrm{~Pa}, z=0.68$, and maximum Hertzian pressures of 2,4 , or $8 \mathrm{GPa}$ leads to viscosities up to the order of $1,10^{+5}$, and $10^{+15} \mathrm{GPa} \cdot \mathrm{s}$, respectively. These high values suggest the question: "What is the physical relevance of the presented results?"

More so since the viscous shear stresses in the fluid and at the fluid-solid contact boundaries also become extremely large. To illustrate this, note that

$$
\eta \partial_{y} v \simeq \eta v_{-} / h
$$

where $y$ is the coordinate across the fluid film, $v$ the velocity along the film and $v_{-}$the difference between the velocities of the surfaces. Evaluating (11) for $v_{-}=1 \mathrm{~m} / \mathrm{s}, \eta=1 \mathrm{GPa} \cdot \mathrm{s}$, and $h=0.1$ micron gives a shear stress of approximately $10^{+7}$ $\mathrm{GPa}$. It is unnecessary to say that this (tangential) loading is much larger than the pressure. Consequently, the deformation of the surfaces caused by the shear stresses cannot be neglected. A detailed analysis of the effect of the viscous stresses can be found in [22] or in a forth-comming paper [23]. In brief, the deformation caused by the shear stresses can be neglected compared to the deformation due to the pressure if $\eta v_{-}=\mathcal{O}\left(q^{2}\right) w$, where $q$ denotes the quotient of the characteristic height and the characteristic length of the fluid domain.

\section{References}

22 Verstappen, R., "Elastohydrodynamic Lubrication: A Dynamic Variation Method," Ph.D. theses, University of Twente, Enschede, The Netherlands, 1989.

23 Verstappen, R., "A First Study of Shear Stress at an ElastoHydrodynamically Lubricated Contact," submitted to Int. J. Engng. Sci.

\section{Authors' Closure}

The authors wish to thank Dr. Verstappen for his careful reading of the paper and would like to make the following remarks:

- The authors agree with the discusser that a solution showing

${ }^{1}$ Department Applied Mathematics, University of Twente, 7500 AE Enschede The Netherlands

* Copies are available free; address the discusser. significant pressure variations at the scale of a few $\AA$ ngström would, at least locally, be in contradiction with the assumption that the fluid behaves as a continuum on which Reynolds equation is based. However, the situation depicted by the discusser is somewhat exaggerated. In practical bearing applications the half width of the Hertzian contact varies from 0.1 to $0.5 \mathrm{~mm}$. The size of the calculational domain is a number of times this half width and in most situations it is at least $O(1) \mathrm{mm}$. Assuming the most extreme situation of $10^{5}$ nodal points on the full domain the resulting mesh size is still not "a few" ^̊ngström.

This discussion on the physical relevance of the solution of the differential problem should, in our opinion, be viewed separately from the subject of accurate numerical solution of the problem. In our situation, the solution of the discretized equation is an $O(h)$ approximation of the solution of the continuous differential problem. Hence, in regions of large gradients, at least locally, a sufficiently small mesh size is required. This means that, using uniform grids, a relatively large number of nodes is needed. One might argue that a similar accuracy can be obtained using less nodes and a non-uniform grid. That argument however, does not hold in case of rough surfaces where only to describe the roughness profile already a considerable number of nodes will be required.

Nevertheless, solving the smooth surface stationary line contact problem with $O\left(10^{5}\right)$ nodal points gives results which are far more accurate than needed for practical applications. This large number of nodes should be seen as a demonstration that the presented techniques provide a solid basis for a fast solver of the point contact problem where such large numbers of nodes will be needed especially if surface roughness effects should be taken into account.

- In the paper it is explicitly stated that the solutions for extreme high loads such as $8.0 \mathrm{GPa}$ are of little practical importance. In addition to the unrealistically high viscosities mentioned by the discusser one could for example mention that in case of steel surfaces beyond some $3.0 \mathrm{GPa}$ plastic deformation will occur which is not taken into account in the model. Besides, at such high pressures the lubricant will most likely behave as a solid instead of a fluid.

To investigate the effects of surface roughness on pressure profile and film thickness in both stationary and transient situations will require a very stable algorithm. The extremely high loaded situations included in the paper should therefore be seen as a demonstration that the presented algorithm provides the possibility to carry out such studies. Another reason to include these situations was to demonstrate that solutions do exist. Hence, the problems with respect to the solution of highly loaded situations reported in the past were numerical problems.

- As far as shear stress calculations are concerned it is well known that, the use of the model should be limited to no slip conditions. When slip occurs, the model predicts unrealistically high shear stresses and coefficients of friction. According to the discusser, tangential deformation should be included in the model. If the purpose is to accurately predict shear stresses and coefficients of friction in practical situations the authors would recommend to take into account the effect of shear heating instead. The viscosity of the lubricant decreases significantly with increasing temperature and the resulting shear stresses will be small compared to the maximum pressure in the contact, e.g. [22].

\section{Additional Reference}

22 Ten Napel, W. E., Klein Meuleman, P., Lubrecht, A. A., Houpert, L., and Bosma, R., 1985, "Traction in Elastohydrodynamic Lubrication at Very High Contact Pressures," Proc. 4th. European Tribology Conf. Eurotrib 1985, Lyon, France. 